\title{
Polarization and Spectral Information Jointly Utilization in Targets Classification under Different Weather Conditions
}

\author{
Chen Chao, Zhao Yong-qiang, Liu Dan, Pan Quan, Cheng Yong-mei \\ Northwestern Polytechnical University, Xi'an, China \\ chunchaaonwpu@gmail.com
}

\begin{abstract}
Although polarization and spectral information utilization has been received great attention with the sensor and detection technology advance, few results are showed to jointly utilize both of this information in targets classification. Polarization and spectral information reveals two different aspects of one single target, and therefore, if both of information is properly used, good performance would be achieved in the classification. In this paper, polarization and spectral information based on imagery is firstly acquired by spectropolarimeter imaging system. And then features that can represent polarization and spectral information, namely reflectance spectrum and degree of polarization spectrum, are extracted from imagery acquired respectively. As our built spectropolarimeter imaging system contains 33 bands at the range from 400 to $720 \mathrm{~nm}$, we proposed a custom band selection scheme which calculates the Euclidean distance of these two extracted features at each band, and select the bands which are respect to the former larger distances to achieve dimensional reduction. The reduced two features are inputted to Support Vector Machines respectively, and the degrees of membership belongs to each classes are assigned. Finally, we integrate these two features through fusion in the decision level using D-S theory. To highlight the advantages of jointly utilization of polarization and spectral information, classification results based on digital number $(D N)$ value and any one single feature are compared to. And to prove the invariance of extracted features to weather conditions, we test the proposed jointly classification algorithm under two different weather conditions. The results based on the proposed method outperform the other three, and the advantage is much more evident in the cloudy weather.
\end{abstract}

Keywords- polarization; reflectance; band selection scheme; classification; different weather

\section{INTRODUCTION}

As sensing technology advances, more and more information can be extracted from scene of interest. Rich information of targets' surface which varies from spatial, spectral, to polarization can be recorded by imaging spectropolarimeter[1,2,3]. According to the imaging chain theory, by exploiting more information about the scene of interest, better classification performance can be achieved. And therefore, targets classification using this new detection technology has been received great attention during recent years $[1,2,4,5,6,7,8,9,10]$. Polarization information which can be only recorded by imaging spectropolarimeter has the unique advantages, such as the edges, texture, etc. of scene of interest could be enhanced $[5,7,11,12]$. And moreover, polarization characteristics of targets provide the penitential way to identify two targets with similar spectral characteristics, and therefore can be used to distinguish camouflage targets in clutter backgrounds $[13,14]$. As reflectance and degree of polarization spectrum reveal the distinct aspects of a scene and are invariant to the change of imaging intense, it is expected that combining the information will greatly enhance classification performance and behave steadily in different weather conditions.

Degree of polarization $(D o P)$ and reflectance spectrum features with selected bands are inputted into two SVM [15] (Support Vector Machine) classifiers respectively when considering SVM classifier performs well in high dimensions problems. Bands selection are finished according to a new custom scheme which calculates the Euclidean distance of these two features at each wavelength across from 400 to 720 $\mathrm{nm}$ with its interim is $10 \mathrm{~nm}[1,3,6,7]$. With our best knowledge, there is a possibility of classification correction improvement if we combine these two different classifiers. With this idea, final jointly classification result is achieved by information fusion in the Dempster-Shafer (DS) [16] rule in the decision level. To highlight the advantages of jointly utilization of polarization and spectral information, the classification results based on $D N$ value and any one single feature are compared to. And to prove the robustness of extracted features to imaging intense, we test this jointly classification method under sunny and cloudy weather conditions. The results based on jointly classification method outperform the other three, and the advantage is much more evident in the cloudy weather.

The rest of paper is organized as follows. Section II gives the polarization and spectral feature extraction from acquire images. Section III introduces the jointly classification algorithm, and the custom band selection scheme is also included. Experimental results are provided in Section IV, with conclusion and discussion in Section V.

\section{FEATURE EXTRACTION}

When light interacts with the surfaces of targets, the polarization state would be changed due to its roughness, conductivity and so on. Stokes vector $\left[S_{0}, S_{1}, S_{2}, S_{3}\right]^{\mathrm{T}}$ is commonly used to express the light polarization state. The first element $S_{0}$ represents the intensity and it can be expressed by the sum of the value of intensities between horizontal and vertical linearly polarized components, while the second element represents the difference between these two oriented directions. The third element represents the difference between

This work is supported by the National Nature Science Foundation of China, No.60602056 and 60634030, and Aeronautics Foundation of China, No.2007ZC53037. 
linearly polarized components oriented at 45 and 135 degrees. $S_{3}$ is respect to the circular polarization component of light and often is ignored since solar light contains little of this kind. With carefully rotate the mounted polarizer before the reflect light entering the detector; we obtain the Stokes components images.

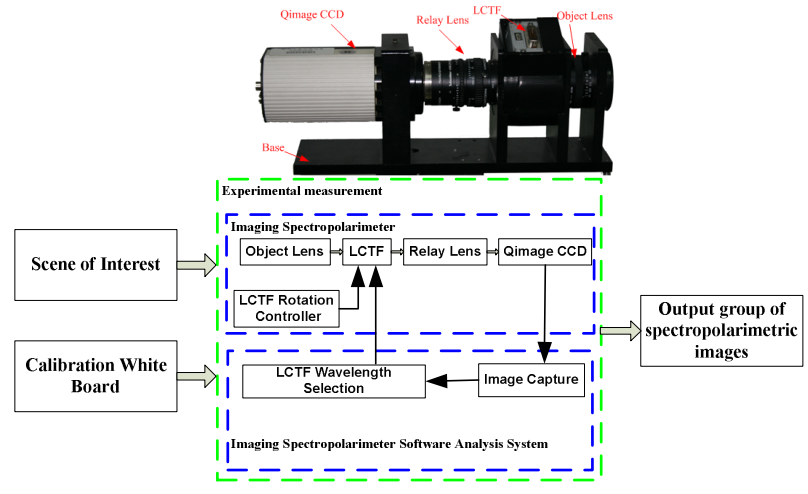

Figure 1. Imaging spectropolarimeter system (Top) and spectropolarimetric data acquisition steps (Bottom)

Imaging spectropolarimeter, which is showed in Fig. 1 (Top), enables us to acquire the spatial, spectral, polarization information simultaneously. The outdoor scene is lit by solar energy directly from sun and scattered by atmosphere under sunny while the scene is only scattered by atmosphere under cloudy weather. We assume the light reaches the surface of target under sunny and cloudy is with non-polarization state, and meet the Eq.1:

$$
I_{\text {cloudy }}=\alpha I_{\text {sun }}
$$

where $I_{\text {sun }}$ is the total radiance that reaches the surface of scene under sunny while $I_{\text {cloudy }}$ is corresponding to the cloudy. We use calibration white board which is with high reflectance more than $99 \%$ to measure $I_{\text {sun }}$ and $I_{\text {cloudy }}$ indirectly. Experimental measurement is showed in Fig. 1(Bottom).

For scene of interest under sunny weather, we have:

$$
\begin{gathered}
S_{(0, \lambda)}=\frac{1}{2}\left(I_{(0, \lambda)}+I_{(45, \lambda)}+I_{(90, \lambda)}+I_{(135, \lambda)}\right) \\
S_{(1, \lambda)}=I_{(0, \lambda)}-I_{(90, \lambda)} \\
S_{(2, \lambda)}=I_{(45, \lambda)}-I_{(135, \lambda)} \\
\operatorname{DoP}(\lambda)=\frac{\sqrt{S_{(1, \lambda)}^{2}+S_{(2, \lambda)}^{2}}}{S_{(0, \lambda)}} \\
\text { Orient }(\lambda)=\tan ^{-1}\left(\frac{S_{(2, \lambda)}}{S_{(1, \lambda)}}\right) \\
R(\lambda)=\frac{S_{(0, \lambda)}}{I_{\text {sun }}}
\end{gathered}
$$

where $I_{0}, I_{45}, I_{90}, I_{135}$ are the radiance that detector receives with polarization direction of $0,45,90,135$ degrees respectively.
And DoP and Orient are derived parameters. As for scene of interest under cloudy weather condition, we have:

$$
\begin{gathered}
S_{(0, \lambda)}=\frac{1}{2} \alpha\left(I_{(0, \lambda)}+I_{(45, \lambda)}+I_{(90, \lambda)}+I_{(135, \lambda)}\right) \\
S_{(1, \lambda)}=\alpha\left(I_{(0, \lambda)}-I_{(90, \lambda)}\right) \\
S_{(2, \lambda)}=\alpha\left(I_{(45, \lambda)}-I_{(135, \lambda)}\right)
\end{gathered}
$$

We can safely conclude that $R, D o P$ and Orient parameters are independent on the radiance that reaches the surface of scene. And from our previous work [1,3,6,7,8], the parameter Orient would be more likely to be noised. And therefore we select $R, D o P$ as classification features, and then if training sets with $R, D o P$ features are obtained in sunny, they can also be used to classify the same scene under cloudy.

\section{JOINTLY CLASSIFICATION ALGORITHM}

\section{A. Band Selection Scheme}

Imaging spectropolarimeter system records $m$ bands and this would enlarge the computing burden if we use all the bands, and therefore we propose a band selection rule which calculates the Euclidean distance of each bands then selects the bands which are respect to the former larger distances. Assume we have $n$ classes of training samples with $m$ bands. The united features that include both $R(\lambda)$ and $D o P(\lambda)$ are denoted as $\operatorname{sp}(\lambda): \operatorname{sp}(\lambda)=[R(\lambda), \operatorname{DoP}(\lambda)]$.

(1). Obtain $s p(\lambda=1,2, \ldots, m)$ of a number of samples belong to each class $(1,2, . ., n)$, like the $k$ th label with $a$ samples. We average all the $a$ samples to avoid the noise and to make it more representative for the $k$ th training sample. We denote it as $\operatorname{sp}(\lambda, k)=[R(\lambda, k) \operatorname{DoP}(\lambda, k)]$;

(2). There would be $n(n-1) / 2$ distance at each band and we save them in a $\mathrm{n} \times \mathrm{n}$ dist matrix. The matrix must be symmetric and the elements in diagnose are zeros as the distance from ith label sample to jth label sample is the same as the distance from $j$ th label to ith label. The distance is expressed in Eq.11.

(3). Redo step (2) and get dist matrix of all the bands $(\lambda=1,2, \ldots, m)$;

(4). Find the non-zero minimal elements in the each dist matrix and sort them in descend order. We select the former $x$ elements, and return $x$ bands with respect to the selected elements; denote them as $\operatorname{sp}(\lambda, k),(\lambda=1,2 \ldots, x, k=1,2 \ldots, n ; x<m)$;

\section{B. Algorithm}

The flow chart of the proposed jointly classification algorithm is illustrated in Fig. 2. The algorithm mainly includes the manually label the training and testing samples, feature extraction, band selection, degree of membership determination, and classification performance evaluation. The details would be introduced in the following steps:

(1). Input the feature of training samples after band selection operation according to SectionIII-A, $R(\lambda), \quad \operatorname{DoP}(\lambda)$, $(\lambda=1,2 \ldots, x$,$) to the SVM classifiers respectively, and$ determine the models of classifiers;

$$
\operatorname{dist}_{i j}(\lambda)=|\operatorname{sp}(\lambda, i)-s p(\lambda, j)|=\sqrt{(R(\lambda, i)-R(\lambda, j))^{2}+(D o P(\lambda, i)-D o P(\lambda, j))^{2}}
$$


(2). Extract features from unclassified scenes, and input them with selected bands that are determined in (1) into the SVM classifiers;

(3). Obtain the degree of membership of $i$ sample belongs to $j$ label from SVM classifiers respectively, $m_{A}(i, j)$ is respected to the output from SVM classifier using $R(\lambda)$ feature while $m_{B}(i, j)$ is respected to output from SVM classifiers using $\operatorname{DoP}(\lambda)$ feature;

(4). Fusion in the decision level to get the final classification results of $i$ sample using D-S rule;

$$
\hat{j}=\underset{j}{\arg \max }\left(\frac{m_{A}(i, j) m_{B}(i, j)}{\sum_{j=1}^{n} m_{A}(i, j) m_{B}(i, j)}\right)
$$

(5). Performance evaluation: We evaluate the results of classification both in quality and quantity. The ground truth of classification results is manually got and the Confuse Matrix and Kappa coefficient are utilized to finish the performance evaluation

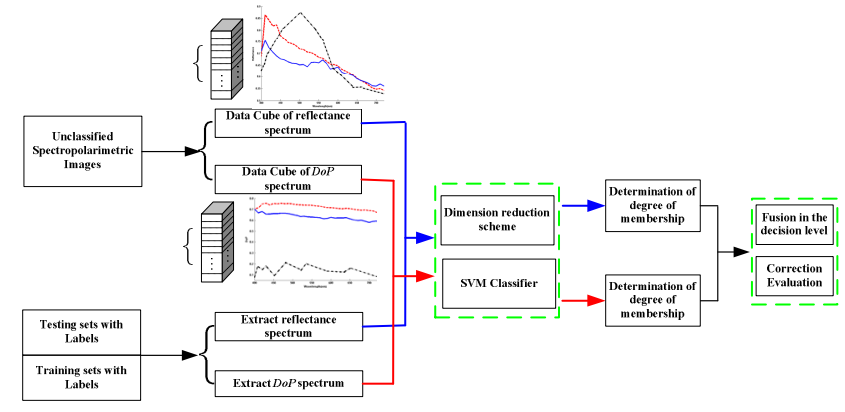

Figure 2. Flow Chart of proposed jointly classification algorithm

\section{EXPERIMENTAL RESULTS}

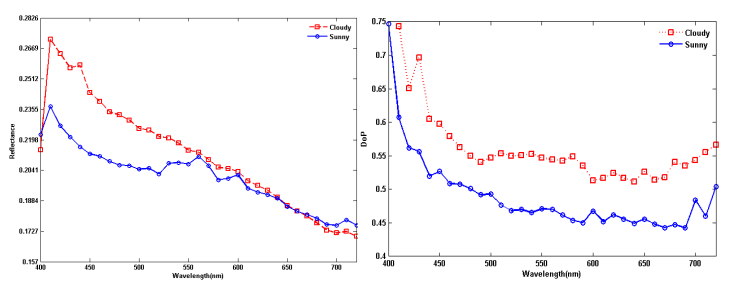

Figure 3. $R(\lambda)(L)$ and $D o P(\lambda)(R)$ black tile under cloudy and sunny condition

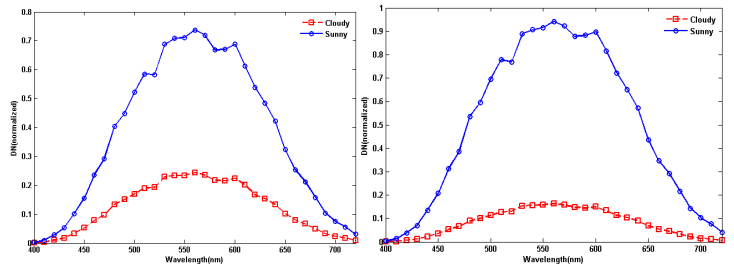

Figure 4. $D N(\lambda)$ of Black-painted board $(L)$ and Black-painted $\mathrm{Al}(R)$ under cloudy and sunny condition

We test the jointly classification in a scene that is composed of five black targets under black cloth background. The training samples are obtained in the sunny weather separately, while the unclassified scene is acquired under sunny and cloudy weather. $R(\lambda)$ and $\operatorname{DoP}(\lambda)$ for black tile under sunny and cloudy are displayed in Fig. 3, while Fig. 4 is respect to $D N(\lambda)$ for other two black-painted targets under sunny and cloudy. Features as $R(\lambda), \operatorname{DoP}(\lambda)$ dynamic a little under sunny and cloudy. However, when comparing to use $D N(\lambda)$ as classification feature directly, features as $R(\lambda), D o P(\lambda)$ are relatively steady under sunny and cloudy. As we can see from Fig. 4, there is no evident different between two black-painted targets. $D N(\lambda)$ as their characteristics is highly affected by response function of $\mathrm{CCD}$, and moreover, they dynamic in a large range under sunny and cloudy, and therefore if training samples are under sunny while unclassified images are under cloudy, then misclassification would be inevitable.

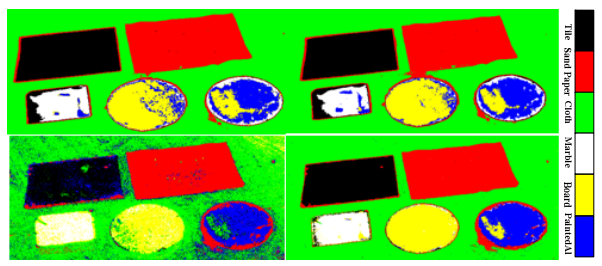

Figure 5. Results of Classification: $D N(\lambda), R(\lambda), D o P(\lambda)$, and jointly classification (From left to right and from top to bottom)

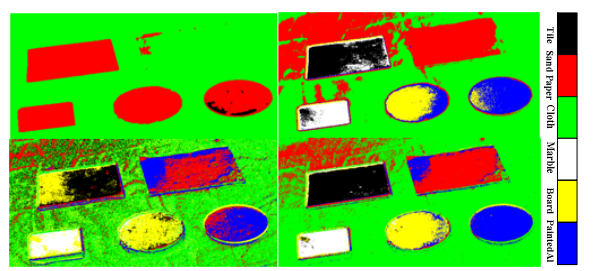

Figure 6. Results of Classification: $D N(\lambda), R(\lambda), D o P(\lambda)$, and jointly classification (From left to right and from top to bottom)

From Fig. 5, we could see that the resluts are all in good performance. This is due to the trainning samples are also got from the same condition. As for the result by using $D N(\lambda)$ feature, some regions near the edges of marble, board and painted $\mathrm{Al}$ are misclassified, and a small continuous region in the painted $\mathrm{Al}$ is misclassified as board. As for the result by using $R(\lambda)$ feature, region near the sand paper and board is misclassified, and the range of the misclassified continuous region in the painted $\mathrm{Al}$ is reduced a little, while for the results by using $\operatorname{DoP}(\lambda)$ feature, the black cloth background is not classified very satisfactory since its $D o P$ is relatively high and similar with the targets. The jointly classification result combines the advantages of result by using $R(\lambda)$ and $D o P(\lambda)$ feature, and is satisfactory except for some misclassification happens in the small region near the edges of Al. Fig. 6 displays the results of classification under cloudy. Since $D N(\lambda)$ feature dynamics dramatically under different weather, and the training samples are from sunny, thus the classification result by using $D N(\lambda)$ feature is rather disappointing. We could only see two classes, the cloth and the sand paper, and the misclassification case is serious. Though result by using $R(\lambda)$ feature is much better than $D N(\lambda)$ feature, they are much worse than the corresponding case under sunny. The left above corner of the scene is almost misclassified as the sand 
paper. As for the result by using $\operatorname{DoP}(\lambda)$ feature, a large region of tile and sandpaper is misclassified, and the cloth background is also not well classified. The jointly classification result, similar as the case under sunny, is the best of all the results under cloudy.

Tab. 1 and 2 give the assessment of classification results quantitatively. We could discover that an increment of $3.71 \%$ in total accurancy and of $4.48 \%$ in Kappa coefficient are achieved in the jointly classification result when comparing to the result by using $D N(\lambda)$ feature, and moreover, the jointly classification result is $5.44 \%$ and $10.12 \%$ improvement in total accuracy and $3.52 \%$ and $12.18 \%$ in Kappa coefficient when comparing to the results by using single $R(\lambda)$ and $D o P(\lambda)$ respectively. The advantage of jointly classification is a lot more evident in the case under cloudy. An increment of $77.88 \%$ in total accuracy and $90.83 \%$ in the Kappa coefficient are obtained in the jointly classification result, compared to the result by using $D N(\lambda)$ feature. An increment of $4.43 \%$ and $21.34 \%$ in total accuracy and $5.44 \%$ and $25.69 \%$ in Kappa coefficient are achieved in the jointly classification result, compared to the results by using single $R(\lambda)$ and $\operatorname{DoP}(\lambda)$ respectively.

Table1 Correction of classification in Scene (Sunny)

\begin{tabular}{ccccccccc}
\hline Feature & BlackTile & SandPaper & Cloth & Marble & Board & PaintedAl & Accuracy & Kappa \\
\hline $\boldsymbol{D} \boldsymbol{N}(\boldsymbol{\lambda})$ & 0.9985 & 0.9990 & 0.9991 & 0.8006 & 0.8234 & 0.7565 & 0.9268 & 0.9099 \\
$\boldsymbol{R}(\lambda)$ & 0.9988 & 0.9994 & 0.9996 & 0.6854 & 0.9391 & 0.6716 & 0.9095 & 0.8895 \\
$\boldsymbol{D o P}(\boldsymbol{\lambda})$ & 0.8912 & 0.9803 & 0.8799 & 0.8056 & 0.7933 & 0.7198 & 0.8627 & 0.8329 \\
Jointly & $\mathbf{0 . 9 9 7 8}$ & $\mathbf{0 . 9 9 9 2}$ & $\mathbf{0 . 9 9 6 6}$ & $\mathbf{0 . 8 9 1 0}$ & $\mathbf{0 . 9 9 1 4}$ & $\mathbf{0 . 8 3 2 5}$ & $\mathbf{0 . 9 6 3 9}$ & $\mathbf{0 . 9 5 4 7}$ \\
\hline \multicolumn{7}{c}{ Table2 Correction of classification } & in Scene (Cloudy) & \\
\hline Feature & BlackTile & SandPaper & Cloth & Marble & Board & PaintedAl & Accuracy & Kappa \\
\hline $\boldsymbol{D} \boldsymbol{N}(\boldsymbol{\lambda})$ & 0 & 0.0214 & 1 & 0 & 0 & 0 & 0.1453 & 0 \\
$\boldsymbol{R}(\boldsymbol{\lambda})$ & 0.9629 & 0.9901 & 0.7992 & 0.7859 & 0.8042 & 0.8631 & 0.8798 & 0.8539 \\
$\boldsymbol{D o P}(\boldsymbol{\lambda})$ & 0.6710 & 0.6153 & 0.6567 & 0.8131 & 0.8508 & 0.7531 & 0.7107 & 0.6514 \\
Jointly & $\mathbf{0 . 9 9 6 2}$ & $\mathbf{0 . 8 0 0 2}$ & $\mathbf{0 . 9 7 4 2}$ & $\mathbf{0 . 9 1 5 0}$ & $\mathbf{0 . 9 1 8 9}$ & $\mathbf{0 . 9 8 0 9}$ & $\mathbf{0 . 9 2 4 1}$ & $\mathbf{0 . 9 0 8 3}$ \\
\hline
\end{tabular}

\section{CONCLUSION AND DISCUSSION}

Few attentions have been taken about the jointly utilization of polarization and spectral information to achieve better classification accuracy. The paper proposes a new method to integrate all the rich information acquired by imaging spectropolarimeter. We also illustrate the selected features dynamic in a tolerant small range under sunny and cloudy weather. A band selection scheme is also studied to reduce the burden of the computing. During our experiments, all the training samples are achieved with almost the same detection geometry as the unclassified scenes separately in order to overcome the effect attributed by nonconsistance of detection geometry between training samples and unclassified scenes. And therefore, the variations would be very little and can be regarded as noise, and the proposed jointly classification algorithm is insensitive to the noise. However, we can predict that the great differences of the detection geometry between training samples and unclassified scenes would largely affect the performance of the classification results. There may be solutions that can overcome this effection and our feature work would focus on them. One possible solution is using some unsupervised classification firstly to obtain the training samples instead of getting them separately, and samples in a scene can be regard as with the same detection geometry.

\section{REFERENCES}

[1] Zhao Yongqiang., Gong Peng, Pan Quan, Object detection by spectropolarimetric imagery fusion[J], IEEE Trans.On Geoscience and Remote sensing 46(10), 3337-3344 (2008).

[2] Frañois Goudail, Patrick Terrier, Yoshitate Takakura, etal Target detection with a liquid-crystall-based passive stokes polarimeter[J], Applied Optics, Vol. 43, Issue 2, pp. 274-282(2004).

[3] Zhao Yong-qiang, Zhang Lei, Pan Quan. Spectropolarimetric imaging for pathological analysis of skin[J]. Applied Optics. 2009, 48(10): D236D246.
[4] Sadjadi, Firooz A., Chun, Cornell S. L. Remote sensing using passive infrared Stokes parameters[J] Optical Engineering, Volume 43, pp. 2283-2291 (2004).

[5] J. Scott Tyo, Dennis L. Goldstein, David B. Chenault, and Joseph A. Shaw Review of passive imaging polarimetry for remote sensing applications[J] Applied Optics, Vol. 45, Issue 22, pp. 5453-5469 (2006)

[6] Zhao Yong-qiang, Qong Peng, Pan Quan Unsupervised spectropolarimetric imagery clustering fusion[J] Appied Remote Sensing, 2009.

[7] Chen Chao, Zhao Yong-qiang, Luo Li etal. Robust materials classification based on multispectral polarimetric BRDF imagery, Proceedings of SPIE, 2009, Beijing.

[8] Zhao Yong-qiang, Pan Quan, Zhang Hong-cai Material Classification Based on Multi-band Polarimetric Images Fusion[C] Proceedings of SPIE, 2006

[9] Wolf, L. B., Polarization-based material classification from specular reflection[J], IEEE Trans. On Pattern Analysis and Machine Learning 12(11), 1059-1071 (1990).

[10] Sadjadi F A, Chun C. Passive polarimetric IR target classification[J]. IEEE TRANSACTIONS ON AEROSPACE AND ELECTRONIC SYSTEMS. 2001, 37(2): 740-751.

[11] Zallat, J., Collet, C., and Takakura, Y., Clustering of polarizationencoded images[J], Applied Optics 43(2),283-292 (2004).

[12] Patrick Terrier, Vincent Devlaminck, Jean Michel Charbois Segmentation of rough surfaces using a polarization imaging system[J] JOSA A, Vol. 25, Issue 2, pp. 423-430(2004).

[13] Sumrain, S. and Giakos, G. C., Efficient polarimetric detection of manmade targets, Imaging Systems and Techniques, 2005. IEEE International Workshop on , 20-25 (2005).

[14] Forssell G, Hedborg-Karlsson E. Measurements of polarization properties of camouflaged objects and of the denial of surfaces covered with cenospheres[C] Proc. SPIE 5075, 246 (2003).

[15] Farid Melgani, L.B. Classification of hyperspectral remote sensing images with support vector machines[J] IEEE Transactions on Geoscience and Remote Sensing 2004, 42(8):1778-1790.

[16] Le Hegarat-Mascle, S., Bloch, I., Vidal-Madjar, D. Application of Dempster-Shafer evidence theory to unsupervisedclassification in multisource remote sensing[J] IEEE Transactions on Geoscience and Remote Sensing, 35(4):1018-1031(1997) 